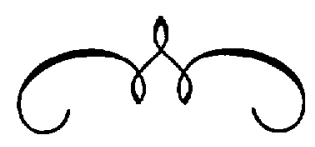

\title{
Proceedings or Transactions?
}

\author{
J. R. PIERCE
}

J. R. Pierce, Editor of the Procendings, has suggested that certain views regarding publications in the TransaCTIONS or Procendings be presented to the membership. It is felt best to present these ideas for comment as prepared by him in their original form.

Questions frequently arise-in Editorial Board discussions and among the reviewers of PROCEEDINGs papers - as to what should be published in the ProceEdirgs and what should be published in the various TransaCTIONS. This statement will not settle the matter, but it does clarify the present situation and suggest future possibilities.

One aspect of the present situation is that of twenty-three Professional Groups of the Institute, twenty-one publish TRANSACTIONS whileonly ten of the twenty-one TRANSACTIONS appear regularly. Thus, in some fields of interest the ProceEdiNGs is the Institute's only facility for publication, and in some it is the only regularly published facility. As long as this situation persists, there must be a certain seeming inconsistency in the publication policies of the Procendings. In some fields the Proceenivgs will endeavor to publish all papers worthy of publication, and in other fields it will publish only a selected few of these papers. It is to be hoped, of course, that neither Transactions nor Proceedings will publish papers which are not worthy of publication.

We hope that this inequity of coverage by the PROCEEDINGs is a temporary matter. While it continues, the Procendings will have to cover some fields in more detail than is perhaps desirable and it may publish too little in the fields of the most active and effective Professional Groups. The remedy is obviously a continued expansion and improvement in the Transactioxs, and effective liaison between the editors of Transactions and the Procendings.

Let us now consider the specific case in which a field is covered both by an active Professional Group with a fine Transactions which appears regularly, and by the Procendings. Among all papers worthy of publication, which should go to the PROCEEDINGS and which to the Transactions? Above all, whatever the decision should be, papers should get into the right journal as promptly as possible. While an initial mistake might in some measure be remedied by republishing in the Procendings a paper which appeared first in the TRANSACTIONS, this is bad, and papers will be reprinted only under the most unusual circumstances. A technical journal is most valuable and most interesting to its readers when its contents are fresh and new. What about simultaneous publication in the Proceedings and in the Traxsactions? This is pointless, for the Procendings reaches all members of the I KE. The problem, then, is to get the papers into the right journal the first time, and promptly.

It is not easy to lay down rules which will cover all papers. There is certainly a place in the Procendings for review papers which summarize recent progress in a field, and for tutorial papers which teach new concepts and techniques of analysis. The ProceEdings asks experts to write such papers.

Most of the material in the Procendings is submitted unsolicited. In trying to lay down rules for reviewing papers, the Editorial Board has said that to appear in the Procendings papers should be important enough to be of general interest (to a reasonable fraction of the Institute's 40,000 members). This has been further interpreted by saying that papers should represent either a contribution of permanent value, or sound work on a topic of great current interest.

In being more specific than this, one can only describe and comment on particular types of papers.
A paper submitted to the Transactions or the Procendings may constitute a sort of review or tutorial paper if it presents a rational approach to a new field quite clearly or completely, so that one unfamiliar or only slightly familiar with the field can use it. Such a paper may or may not contain important new information. If it is really good, it will be suitable for the Procendicigs.

A paper may describe an important new invention or device. If this device has considerable present or potential importance, and if it has real novelty, the membership of the Institute should know about it promptly in a paper of length adequate to explain it clearly. Perhaps the author will want to treat at length many important details which are of interest largely to specialists; he should do so in the Transactions. The Procendings paper should, however, be a real technical paper and not a news item; it should be technically sound and complete enough so that a more detailed discussion can be based on it and can refer to it.

A new experimental result, or new theory, should be treated much as is a new invention or device.

Many papers are concerned with calculations of great importance, about, for example, vacuum tubes, networks, antennas, or solid-state devices. If a calculation exhibits a new and important principle which casts new light on important problems, it is suitable for the Proceedings. If it merely solves a particular difficult problem by ingenious but special methods, it will usually be of interest only to specialists. In such a case, the result, if it is important, can be conveyed to the general IRE membership through the abstracts of Traxsactioss papers which appear in the Procendixgs, or through a letter to appear as Correspondence.

In all cases, we should ask, "Should people outside a particular Professional Group be told about the contents of the paper? Why should they be told? How much should they be told to make the telling a real technical communication and not a news note?"

Where should an author send his paper? If he believes that he has something to say to the entire IRE membership, he should send it directly to the ProceEoIngs. If he is addressing only experts in his field, he should send it to the TRAxsactions. To assure the promptest publication the author should make the right choice.

Sometimes the author makes the wrong choice. It is up to the reviewers to be alert about this, and to recommend to the ProceenINGS or TRANSACTIONS a paper which they feel has been misdirected. This makes some sort of liaison desirable. Several Professional Groups have named one or more of their members to act as one of the three ProceEdings reviewers on all papers in the field of their Group. This makes available at the time of the review for the ProceEdings the opinion of a representative of the Professional Group.

The relationship between the Transactions and Procendixgs must evolve. During this evolution, it is important that at each stage both the Traxsactions and ProceEdings publish promptly interesting and worthwhile material. The material must be worthwhile in each case, and perhaps the chief distinction must be between what is interesting to a large and heterogeneous group and what is interesting to a specialized segment. 